\title{
Effect of complex landscape geometry on the invasive species spread: invasion with stepping stones
}

\author{
Weam Alharbi \& Sergei Petrovskii ${ }^{1}$ \\ Department of Mathematics, University of Leicester, \\ University Road, Leicester LE1 7RH, U.K.
}

\begin{abstract}
Spatial proliferation of invasive species often causes serious damage to agriculture, ecology and environment. Evaluation of the extent of the area potentially invadable by an alien species is an important problem. Landscape features that reduces dispersal space to narrow corridors can make some areas inaccessible to the invading species. On the other hand, the existence of stepping stones - small areas or 'patches' with better environmental conditions - is known to assist species spread. How an interplay between these factors can affect the invasion success remains unclear. In this paper, we address this question theoretically using a mechanistic model of population dynamics. Such models have been generally successful in predicting the rate and pattern of invasive spread; however, they usually consider the spread in an unbounded, uniform space hence ignoring the complex geometry of a real landscape. In contrast, here we consider a reaction-diffusion model in a domain of a complex shape combining corridors and stepping stones. We show that the invasion success depends on a subtle interplay between the stepping stone size, location and the strength of the Allee effect inside. In particular, for a stepping stone of a small size, there is only a narrow range of locations where it can unblock the otherwise impassable corridor.
\end{abstract}

Keywords: invasion blocking; dispersal corridors; diffusion-reaction model; Allee effect

\footnotetext{
${ }^{1}$ Corresponding author. Email: sp237@le.ac.uk
} 


\section{Introduction}

Invasive species are known to be a major problem in ecology resulting in significant biodiversity loss worldwide [25, 78] and causing serious problems for agriculture, forestry and fishery worth billions of dollars annualy [43, 70]. For these reasons, biological invasion has been a major focus of intense emprical and theoretical research for several decades $[20,73,92]$ with the number of papers growing with time nearly exponentially [74]. In particular, a variety of invasion scenarios have been identified [37, 39, 69, 85] and considerable progress has been made in understanding how various factors can affect the rate and pattern of species spread into space [57]. Having that said, due to the high complexity of the phenomenon (cf. [77]) many questions remain open and many issues are lacking clarity [14].

Factors affecting the spread depend on the spatial and temporal scales at which the phenomenon is considered. In particular, this bears upon the role of the environmental heterogeneity. Much of the theoretical research has been concerned with invasive spread occurring either in large spatially-uniform areas or in an environment with a small-scale heterogeneity (e.g. in the form of environmental noise). Relevant modelling frameworks are given by reaction-diffusion equations $[36,55,64,67,85,86,90]$, stochastic models [54, $56]$ including stochastic reaction-diffusion equations [28, 58, 89], and integral-difference equations [46, 48, 63].

Whichever particular model is used to study the invasive spread, it is usually considered in an unbounded space hence neglecting the effect of domain boundaries. However, this assumption of the environmental heterogeneity is restricted to a certain spatial scale and ceases to be valid when the spreading alien population approaches the limits of the corresponding geographic range. On a larger scale, invasive spread can be considered in a fragmented, 'discrete' environment where disjoint habitats are connected by dispersal; relevant modelling framework includes coupled map lattices [16, 41, 59] and networks [82].

Interestingly, the intermediate case of environmental heterogeneity occurring on a "not too large' spatial scale, e.g. where the typical spatial size of the heterogeneity is the same as the typical scale of the spreading alien population (e.g. as is given by the variance of the dispersal kernel) has been rarely considered (but see [30, 31]). In particular, the effect of habitat's boundaries on the invasive species spread remains poorly understood, especially in case of a nontrivial landscape geometry. Meanwhile, in a somewhat more general ecological context, the importance of the boundaries and the habitats shape for the population dynamics is widely recognized [8]. In our recent work, we addressed the above problem by considering invasive spread in a dumbbell-shaped domain where two large habitats are connected by a narrow passage or corridor and showed that the effect of the corridor can be nontrivial and counterintuitive [3]. In this paper, we consider a spatial system with a more complex geometry where the corridor is not uniform but includes a patch with different properties. In case the environment inside the patch is more favourable for the population (e.g. resulting in a higher growth rate or lower mortality 
rate) than the rest of the corridor, such a patch can play the role of a 'stepping stone' for the invading population [7, 40, 42, 79].

The main goal of this study is to reveal the relation between the ecologically meaningful factors such as the stepping stone patch size, location, and the environment quality inside the patch that can hamper or promote invasive species spread in a complex landscape. The paper is organized as follows. In Section 2, we introduce our modelling framework and consider the effect of stepping stones in a simple one-dimensional system. In Section 3 , we consider the effect of stepping stones in a hypothetical two-dimensional H-shaped domain. Last section provide discussion and conclusions.

\section{Stepping stone in $1 \mathrm{D}$ case}

In order to make an inceptive insight into some generic properties of invasive spread with stepping stones, we begin with simple one-dimensional (1D) system.

\section{$2.1 \quad$ Model}

We consider a single-species reaction-diffusion model where the local population growth is hampered by the Allee effect. In the 1D space, the model is given by the following equation $[55,62,91]$ :

$$
\frac{\partial u(x, t)}{\partial t}=\kappa \frac{\partial^{2} u}{\partial x^{2}}+\gamma u(K-u)(u-b(x)),
$$

where $u$ is the population density of the alien species at position $x$ and time $t, \kappa$ is the diffusion coefficient, $\gamma$ is a coefficient quantifying the population growth rate, $K$ is the carrying capacity and $b$ is the measure of the strength of the Allee effect to which we will refer as the Allee threshold [55].

The number of parameters in Eq. (2.1) can be decreased by choosing dimensionless variables:

$$
\tilde{u}=\frac{u}{K}, \quad \tilde{x}=x \sqrt{\frac{\gamma K^{2}}{\kappa}}, \quad \tilde{t}=t \gamma K^{2} .
$$

Equation (2.1) then takes the following form:

$$
\frac{\partial u(x, t)}{\partial t}=\frac{\partial^{2} u}{\partial x^{2}}+\hat{\gamma} u(1-u)(u-\beta(x)),
$$

where tildes are omitted for the sake of notation simplicity. Here $\beta=b / K$ is the normalized, dimensionless Allee threshold, and $\hat{\gamma}$ is a dimensionless coefficient. In the rest of this section, $\hat{\gamma}=1$; we keep this factor for convenience, its meaning will become clear in Section 3.3. The Allee effect is called weak for $-1<\beta \leq 0$ and strong for $0<\beta<1$ [91]. In the corresponding nonspatial case, i.e. Eq. (2.2) without the diffusion term, the system has three steady states in the case of strong Allee effect (stable states $u=0$ and 
$u=1$ and unstable $u=b$ ) but only two in case of the weak Allee effect (stable $u=1$ and unstable $u=0)$.

In case the population dynamics is considered in a spatially uniform environment, $\beta$ is a constant parameter. In an unbounded space, the generic solution of Eq. (2.2) relevant to biological invasion (i.e. for appropriately chosen initial conditions) is then a travelling population front $[55,62]$ connecting the lower stable steady state $u=0$ to the upper stable steady state $u=1$; see Fig. 1 . Invasion is successful (the front propagates towards the areas where the invasive species is absent, i.e. to the right in Fig. 1) if $\beta<0.5$ and invasion fails (the population front retreats, i.e. propagates to the left in Fig. 1) if $0.5<\beta<1$ [62]. Below we will refer to the case $\beta>0.5$ as a 'very strong' Allee effect. In case the spatial domain is bounded but sufficiently large, strictly speaking the travelling front solution of Eq. (2.2) is not valid any more but it provides "an intermediate asymptotics" [6]: a good approximation of the actual solution (for relevant initial and boundary conditions) for the time range when the front is sufficiently far away from the domain boundaries.

In the present study, in order to account for the effect of environmental heterogeneity, we consider $\beta$ to be a function of space. More specifically, we consider the system where the spatial domain is split into a succession of several subdomains or habitats - say, Habitats A, B, C, D and E (see Fig. 2) that differ by their quality. We assume that the quality of the environment is quantified by a single parameter, i.e. by the strength of the Allee effect. Correspondingly, Habitat $X$ is described by the Allee threshold $\beta_{X}$ and by its length $L_{X}, X=A, \ldots, E$, where $L_{A}+L_{B}+L_{C}+L_{D}+L_{E}=L$ and $L$ is the overall length of the domain. We mention it here that a similar spatial design is often seen in studies on biological invasions in real-world systems, e.g. see [5, 9, 33].

In our choice of values $\beta_{X}$, we focus on the situation where the invasive species in its spread has approached a 'bad' area with unfavorable conditions (environment of poor

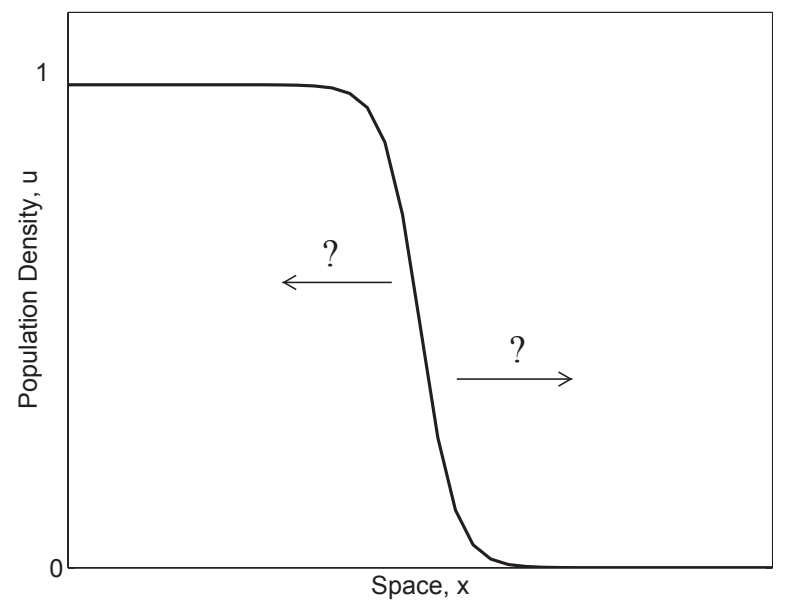

Figure 1: Travelling population front as the generic solution of Eq. (2.2) in a uniform unbounded space. The direction of the front propagation (shown by the arrows) depends on the value of $\beta$, as is emphasized by the question marks: the front propagates to the right for $\beta<0.5$ (successful invasive spread) and to the left for $0.5<\beta<1$ (species retreats, invasion failure). 
(a)

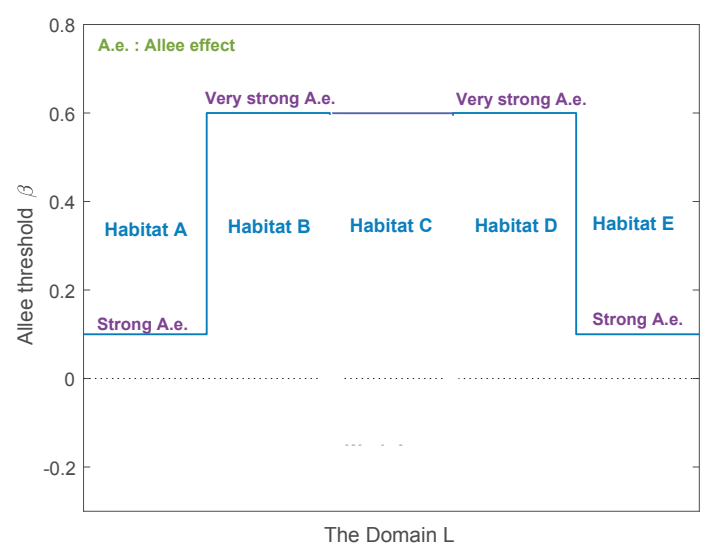

(b)

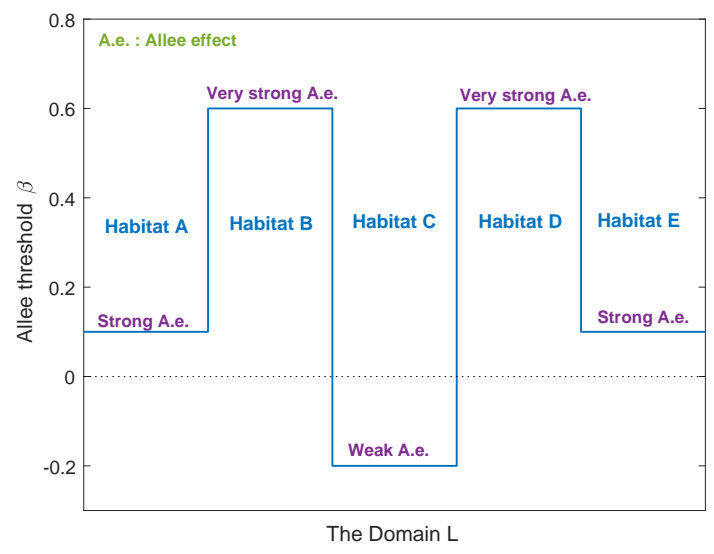

Figure 2: Sketch of the domain structure: (a) a wide 'barrier' of unfavourable environment (with a very strong Allee effect, $0.5<\beta<1$ ), as given by combined Habitats A \& B \& $\mathrm{C}$, separates the invaded Habitat A from potentially invadable but inaccessible Habitat $\mathrm{E}$ behind the barrier; (b) the same as in (a) but now with a 'stepping stone' of favourable environment (Habitat $\mathrm{C}$ ) in the middle of the barrier.

quality) where the Allee effect is very strong, $0.5<\beta<1$; see combined habitats $\mathrm{B}$, $\mathrm{C}$ and $\mathrm{D}$ in Fig. 2a. In case the length of this unfavorable area is small, i.e. smaller than a certain critical value [66], the propagating population front will eventually overcome the bad area (sometimes subject to a considerable delay [65]) and continue spreading at the other side, i.e. into favourable Habitat E. In case the length of the unfavorable area is larger than the critical value, the propagating population front will stop at the interface between Habitats $\mathrm{A}$ and $\mathrm{B}$, even that the environment behind this barrier is favourable again [66]. The question that we are concerned here with is how this situation may change if there is a domain with favorable conditions (e.g. a weak Allee effect, $\beta<0$ ) inside the unfavorable area, see Habitat $\mathrm{C}$ in Fig. 2b, to which we refer as the 'stepping stone' of invasion.

We assume that the invasive species spreads from left to right; correspondingly, for the initial condition, we consider the situation that the invasive species is present at its carrying capacity in Habitat A but absent from all other habitats, that is

$$
u(x, 0)=1 \quad \text { for } \quad 0 \leq x \leq L_{A}, \quad u(x, 0)=0 \quad \text { for } \quad L_{A}<x<L .
$$

For the conditions at the external domain boundaries, we consider the zero-flux Neumann condition at the left-hand side boundary and the Dirichlet-type zero function at the right-hand side boundary:

$$
\frac{\partial u(0, t)}{\partial x}=0, \quad u(L, t)=0
$$




\subsection{Simulation results}

Equation (2.2) with $\beta(x)$ defined as a piecewise-constant function (cf. Fig. 2) was solved by finite differences ${ }^{2}$. The steps of the numerical grid were chosen sufficiently small to avoid numerical artifacts and it was checked that the results do not change with a further decrease of the grid step sizes.

Having performed extensive simulations for various parameter values, we have identified three essentially different invasion regimes that are shown schematically in Fig. 3. In Case 1, the population stops at the boundary between Habitats A and B. The population is at approximately its carrying capacity in A but fast decays to zero outside of Habitat A. In Case 2, the population spills over to Habitat $\mathrm{C}$ and establishes there at the population density approximately equal to the carrying capacity, but is blocked at the boundary between Habitats $\mathrm{C}$ and $\mathrm{D}$, hence never spilling over to Habitat E (except for an exponentially thin tail). In Case 3, the population first overcomes unfavorable Habitat $\mathrm{B}$ and establishes itself in favorable Habitat C (stepping stone) and then eventually spills over to favorable Habitat E. We mention here that, depending on parameter values there may be minor variation in the above scenarios, e.g. the density profile shown in Case 2 may not have the trough or, on the contrary, the density profile shown in Case 3 may have a trough located in Habitat D. Since our main goal is to distinguish between the successful invasion (population overcomes the bad area) and the invasion failure (popu-

\footnotetext{
${ }^{2}$ Equation (2.2) was solved in each of the habitats separately and the solutions were then matched together using the conditions of continuity of the function and of the flux at the boundaries separating the habitats.
}

(a) Case 1

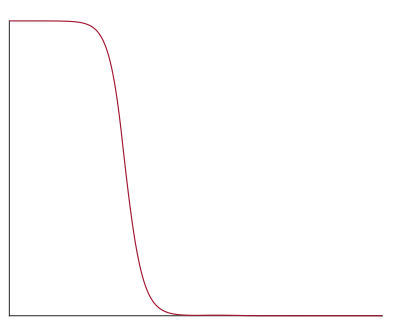

(b) Case 2

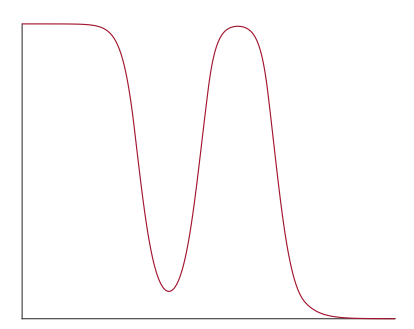

(c) Case 3

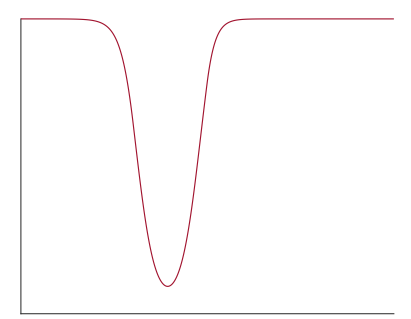

Figure 3: Invasion scenarios in the hypothetical landscape with a 'stepping stone' inside the unfavourable area; the solid red curve shows the population density. (a) Invading population is blocked at the interface between habitats $\mathrm{A}$ and $\mathrm{B}$. This happens if either $L_{B}$ is sufficiently large or the Allee effect is very strong in Habitat B (i.e. $\beta_{B}$ is close to one) and the conditions are not sufficiently good in the stepping stone Habitat $\mathrm{C}$ ( $\beta_{C}$ is not small enough). (b) Invading population passes through unfavourable Habitat $\mathrm{B}$ and establishes in favourable Habitat $\mathrm{C}$ but cannot spill over to Habitat E. This happens if either $L_{D}$ and/or $\beta_{D}$ are sufficiently large. (c) Invading population spills over the gap to Habitat E. This happens when neither of $L_{D}$ or $\beta_{D}$ is too large. 
lation is blocked by the bad area), here we do not pay much attention to those relatively minor details of the population profile.

We consider $L_{C}$ and $\beta_{C}$ as controlling parameters of the problem and make a more detailed insight into how the properties of the stepping stone Habitat $\mathrm{C}$ affect the dynamics of the invasive spread. Our specific goal is to reveal for what parameter values the blocked invasion (Case 1 in Fig. 3) changes to successful invasion (Case 3 in Fig. 3). Apparently, the invading population cannot spill over to Habitat E behind the barrier before establishing in Habitat $\mathrm{C}$ first. Indeed, a necessary condition for the population to spread into Habitat $\mathrm{E}$ is that it should exceed the Allee threshold $\beta_{E}$ at the entrance to the habitat, i.e. at the boundary between Habitats D and E. Correspondingly, it means that the tail of the population density arriving to Habitat E from the left should be larger than $\beta_{E}$. However, it is hardly possible if the population density is low in Habitat $\mathrm{C}$. Therefore, one point to understand is when Case 1 changes to Case 2.

Note that, since the Allee effect in Habitat $\mathrm{C}$ is assumed to be weak $\left(\beta_{C}<0\right)$, the population growth is positive there, even for a very small population density. Correspondingly, the decay in the density profile shown in Fig. 3a is not necessarily monotonous but can have a hump at the location of Habitat C. The height of the hump depends on the parameters of Habitats B, C and D (the stepping stone and the two adjoining habitats), in particular on the length $L_{C}$. For a small $L_{C}$, the hump is hardly visible, but it grows with an increase in $L_{C}$. Once $L_{C}$ exceeds a certain critical value, the height of the hump jumps to a much bigger value with the population density in the middle of Habitat $\mathrm{C}$ being approximately equal to the carrying capacity: Case 1 changes to Case 2 .

The question remains as to what is the relation between the parameters of the stepping stone that distinguish between the invasion success and invasion failure. This has been addressed through extensive numerical simulations where we fix other parameters as $L_{A}=$ $L_{B}=15, \beta_{A}=\beta_{E}=0.3, \beta_{B}=\beta_{D}=0.6$ and $L_{D}=4$ and vary $\beta_{C}$ and $L_{C}$ in a broad range of values. The corresponding structure of the parameter plane $\left(\beta_{C}, L_{C}\right)$ is shown in Fig. 4a. It is readily seen that, for Habitat $\mathrm{C}$ to be invaded, either its length must be sufficiently large or the Allee effect must be sufficiently weak (i.e. the quality of the environment should be sufficiently high).

For the parameter values of Fig. 4a, the population cannot proliferate to Habitat E as it is blocked by unfavourable Habitat D. Hence the expectation is that, provided the population establishes in Habitat $\mathrm{C}$, the success of its further spread depends on the parameters of Habitat $\mathrm{D}$, in particular on its length. Intuitively, one can expect that the spread can only be blocked if $L_{D}$ is sufficiently large. In case $L_{D}$ is not large enough, successful establishment in Habitat $\mathrm{C}$ should inevitably lead to a successful spread to Habitat E, i.e. Case 2 would change to Case 3. This intuitive expectation is confirmed by simulation results. Figure $4 \mathrm{~b}$ shows parameter plane $\left(\beta_{C}, L_{C}\right)$ obtained for a smaller value $L_{D}=3$, other parameters being the same as in Fig. 4a. It is readily seen that for this value of $L_{D}$ Case 2 cannot happen: whenever the population spreads to Habitat $\mathrm{C}$, it spreads to Habitat E too. 
(a)

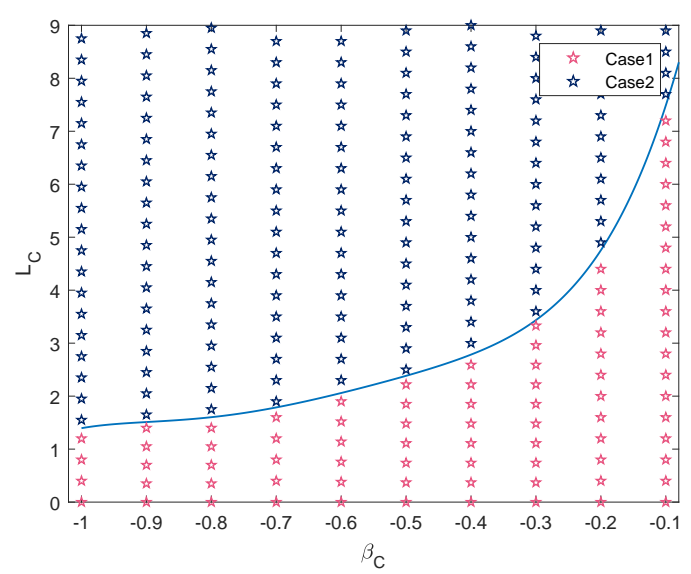

(b)

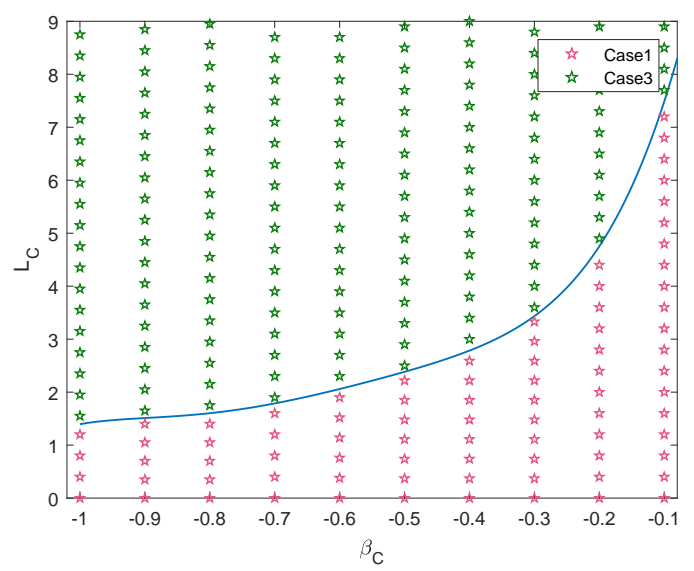

Figure 4: Map in parameter plane $\left(\beta_{C}, L_{C}\right)$ where the domains above and below the curve correspond to different invasion scenarios obtained for (a) $L_{D}=4$ and (b) $L_{D}=3$. Other parameters are $\beta_{A, E}=0.3, \beta_{B, D}=0.6$ and $L_{A}=L_{B}=15$.

Numerical simulations performed for various values of $L_{D}$ reveal that, for other parameter values fixed as above, the critical length of Habitat D is approximately 3.7. However, this value is likely to depend on the strength of the Allee effect in the stepping-stone Habitat C. In order to make a more detailed look into this issue, we now fix the length of Habitat $\mathrm{C}$ along with other parameters and vary the length of Habitat D. Figure 5 shows the structure of parameter plane $\left(\beta_{C}, L_{D}\right)$. It is readily seen that the dynamics does depend on the strength of the Allee effect, albeit slightly, the main controlling parameter being the length of the bad area $L_{D}$.

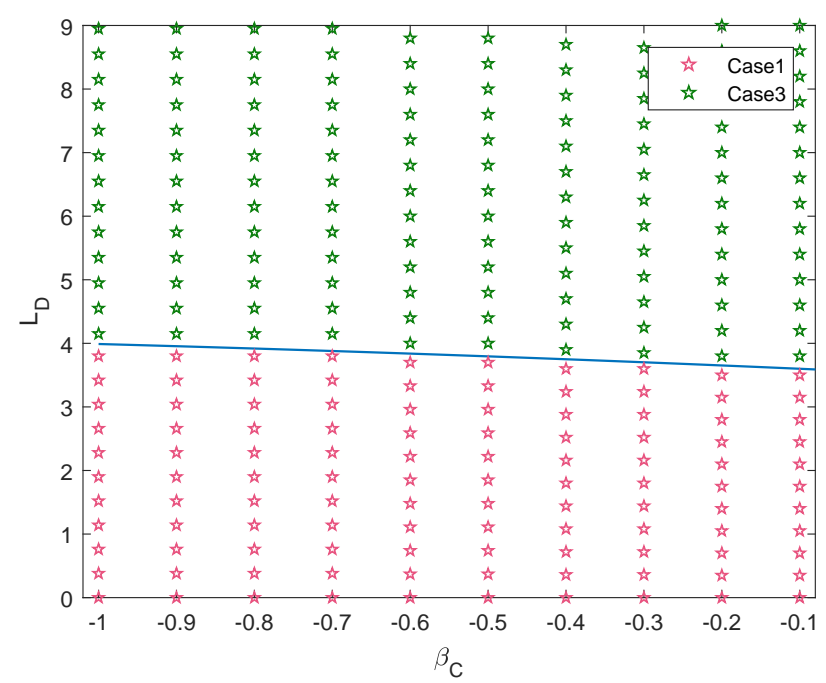

Figure 5: Critical size of Habitat D as a function of the strength of the Allee effect in stepping stone Habitat C (solid curve). For parameters above the curve the population spreads to Habitat E, for parameters below the curve the spread is blocked by bad Habitat D. 


\section{$3 \quad$ Stepping stones in $2 \mathrm{D}$ case}

The results of the previous section provides a first insight into the problem, in particular showing that the outcome of the invasive spread (i.e. success or failure) depends on a subtle interplay between the parameters of the bad area and those of the stepping stone patch, in particular its location and the strength of the Allee effect inside the patch. However, one feature of the dynamics that is completely missed out by the 1D system is the possibility of the spreading population to overcome the bad area by going around it. In this section, we are going to address this issue by considering the problem in a more appropriate 2D layout of the model.

In the $2 \mathrm{D}$ space $(x, y)$, the model is given by the following reaction-diffusion equation:

$$
\frac{\partial u(x, y, t)}{\partial t}=\frac{\partial^{2} u}{\partial x^{2}}+\frac{\partial^{2} u}{\partial y^{2}}+u(1-u)(u-\beta(x, y))
$$

(in dimensionless units) where notations have the same meaning as in Section 2.1. We consider an H-shaped domain where two large rectangular sub-domains of the same size say, Habitat 1 and Habitat 2 - are connected by a passage; see Fig. 6 (note that, in order to avoid confusion, we label the habitats differently from the 1D case.) At the boundary of the domain (including the passage boundaries) we use the zero-function Dirichlet type condition; from the ecological perspective, it implies that the areas outside of the H-shaped domain are extremely unfavourable.

Our goal is to consider how the invasion success can be affected by the existence of the stepping stone (cf. Fig. 6) - a patch situated inside either the passage or one of the habitats with more favourable conditions than in the rest of the domain. Inside the patch, we consider the Allee effect to be weak (except for a few clearly stated special

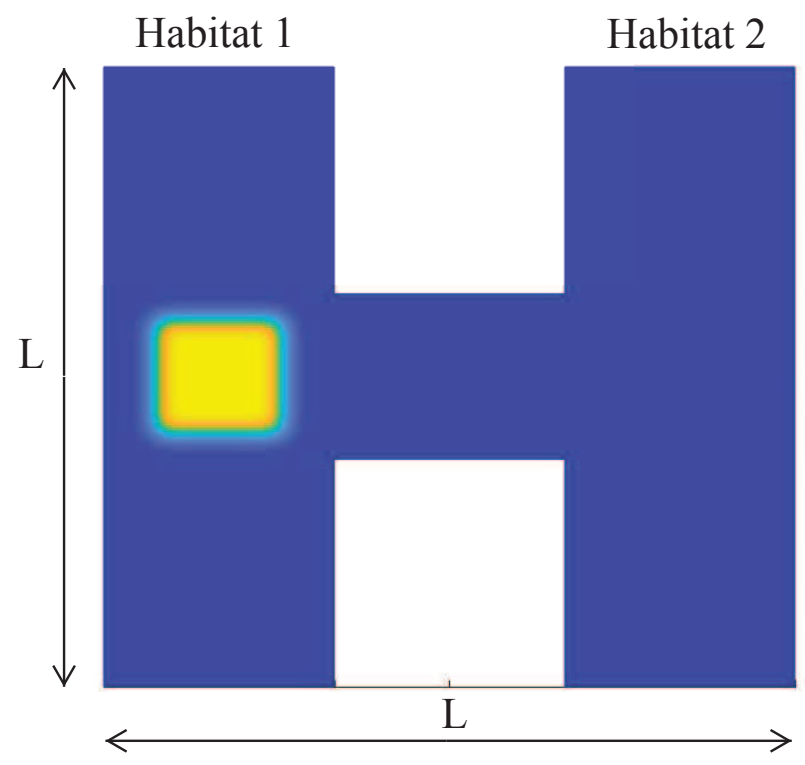

Figure 6: Sketch of the 2D computational domain to study the effect of the favourable patch ('stepping stone', shown by yellow square) on the invasion success. 
cases); elsewhere in the domain, we consider it to be strong. The strength of the Allee effect inside and outside the patch is quantified by the value of the Allee threshold as $\beta_{w}(\leq 0)$ and $\beta_{s}(>0)$, respectively.

The problem is studied by means of extensive numerical simulations. Equation (3.1) is solved numerically by finite differences. In most cases, we used the simple explicit scheme with mesh steps $\triangle x=\triangle y=0.1$ and $\triangle t=0.001$. In all simulations shown below, the overall size of the computational domain is $L=50$. For the value of the Allee threshold, we fix $\beta_{s}=0.1$ but vary $\beta_{w}$ using different values (normally, between 0 and -1 ) in different simulations.

\subsection{Establishment}

We first consider how the existence of a stepping stone can help the establishment of an alien species in a new environment. For this purpose, we consider the situation where the stepping stone is located inside Habitat 1 (see Fig. 6).

Let us begin with the special case where $\beta_{w}=\beta_{s}>0$ which means that, effectively, there is no stepping stone. We consider that the alien species is introduced into a small square-shaped area to which we for convenience refer as 'IC-patch'. We assume that, immediately after the introduction, the alien species is distributed uniformly inside the patch with density $u_{0}$. Correspondingly, the initial conditions for Eq. (3.1) are as follows:

$$
u(x, y, 0)=u_{0} \quad \text { for } \quad(x, y) \in \text { IC-patch, } \quad u(x, y, 0)=0 \quad \text { otherwise. }
$$

It is well known that, in case the population growth is affected by the strong Allee effect, the species introduction into a finite area of a uniform space exhibits criticality. The species persists if and only if the initial population density is sufficiently large, i.e. larger than a certain critical value where the latter depends on the size of IC-patch $[57,67]$. Simulation results obtained for Eqs. (3.1-3.2) in the case $\beta_{w}=\beta_{s}=0.1$ are shown in Fig. 7a. It is readily seen that a smaller IC-patch does require a higher initial population density to ensure the survival of the alien population as the critical patch size is a monotonously decreasing function of the initial density.

A question now arises as to how this situation may change if the place of the species introduction has more favourable environmental conditions than the rest of the domain, i.e. if the IC-patch effectively coincides with the stepping stone. We mention here that, unlike the case of a uniform space, the problem of species establishment in a nonuniform space has been poorly studied. Figure $7 \mathrm{~b}-\mathrm{d}$ shows simulation results obtained for a few different values of the Allee threshold $\beta_{w}$ inside the patch. We observe that the system possesses the properties similar to those shown in Fig. 7a: there exists a critical value of the initial population density, the critical patch size is a decreasing function of of the initial population density. Interestingly, for a sufficiently weak Allee effect inside the patch (cf. Figs. 7c,d), the critical patch size exhibits only a weak dependence on $u_{0}$. 
(a) $\beta_{w}=0.1$

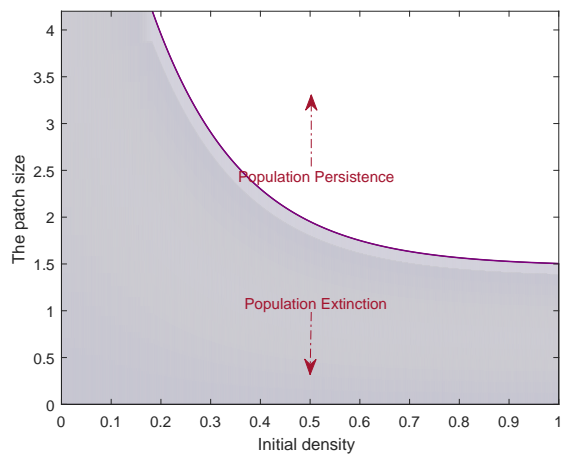

(c) $\beta_{w}=-0.5$

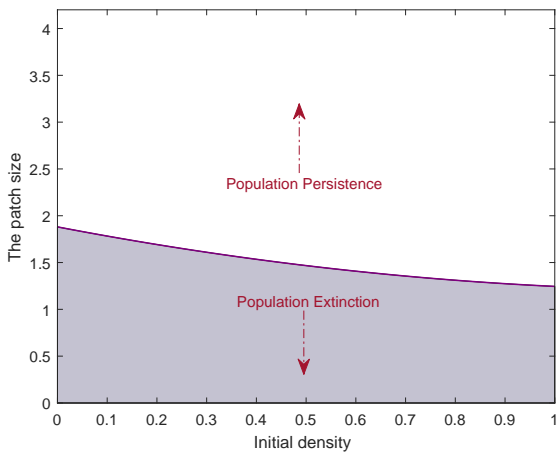

(b) $\beta_{w}=-0.1$

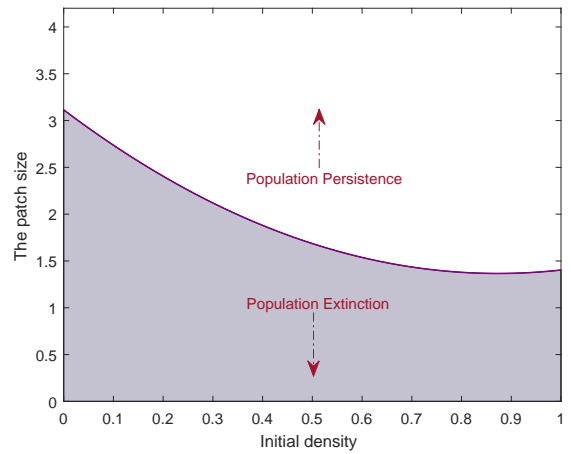

(d) $\beta_{w}=-1$

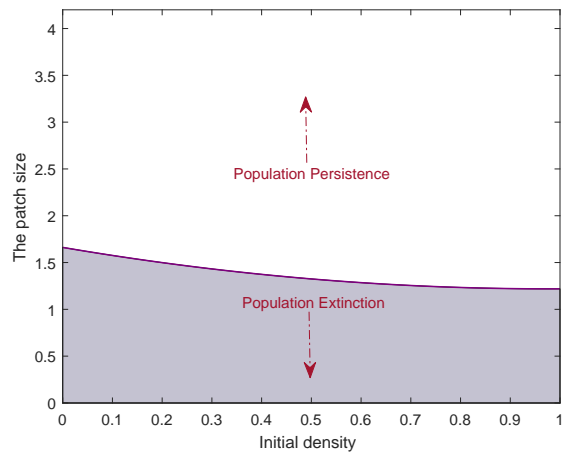

Figure 7: Critical patch size as a function of the initial population density $u_{0}$ for a different strength of the Allee effect inside the stepping stone patch. The Allee effect is strong in (a), weak in (b,c) and absent in (d). In the rest of the domain the Allee effect is strong, $\beta_{s}=0.1$. Parameters of the passage are $($ length, width $)=(12,7.5)$.

To further demonstrate the effect of the stepping stone on species establishment, Fig. 8 shows the dependence of the critical patch size on the strength of the Allee effect inside

(a) $u_{0}=0.1$

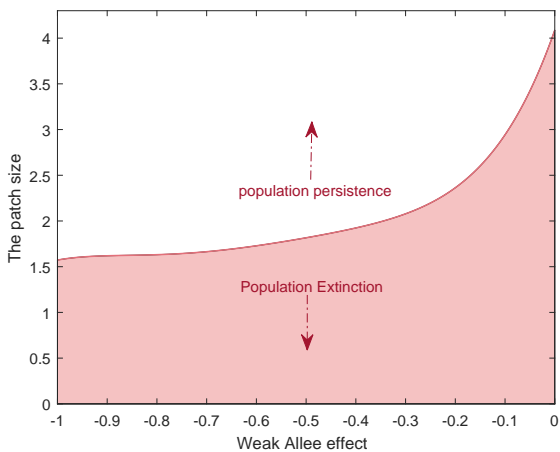

(b) $u_{0}=0.3$

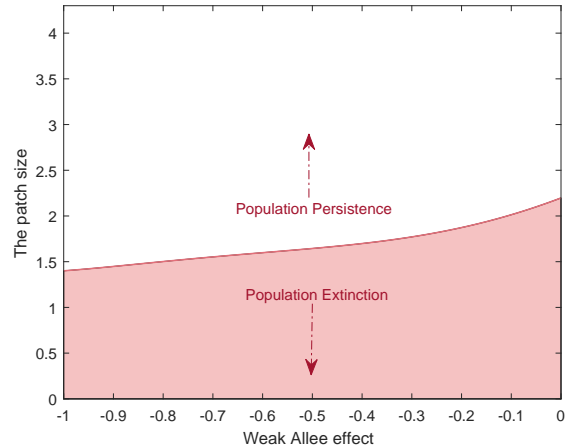

Figure 8: Critical patch size as a function of the strength of the Allee effect inside the stepping stone patch (quantified by $\beta_{w}$ ) obtained for two values of the initial population density. Other parameters are the same as in Fig. 7. 
the patch. We observe that, the weaker the Allee effect is, the smaller the critical patch size is (i.e. the more likely the alien species to persist) and this effect is more prominent for smaller values of the initial population density.

\subsection{Spread through the passage}

In the rest of Section 3, we consider the situation where the alien species has already invaded Habitat 1. Correspondingly, the initial conditions are used as follows:

$$
u(x, y, 0)=1 \quad \text { for } \quad(x, y) \in \text { Habitat } 1, \quad u(x, y, 0)=0 \quad \text { otherwise. }
$$

It has been shown in our previous work that, in case of a uniform environment ( $\beta$ is constant everywhere inside the domain, no stepping stones), the invasion success in the $\mathrm{H}$-shaped geometry is determined by the size of the passage $[2,3]$. The alien population spills over to Habitat 2 if and only if either the width of the passage is sufficiently large or the length is sufficiently small; otherwise it is blocked by the passage. Here we are going to consider the effect of the stepping stone (cf. Fig. 9). Our aim is to reveal the relation between the factors that can affect the invasion success (the spill-over of the alien species to Habitat 2), namely, between the size of the patch, the patch location inside the passage (quantified by the distance $l_{p}$ between the patch and the entrance to the passage from Habitat 1), and the strength of the Allee effect inside the patch.

It was observed in simulations using the model (3.1), (3.3) that, in case of a successful spread, the population spill-over to Habitat 2 is usually well seen by $t=500$ or so. Correspondingly, for all parameter values where invasion failure is seen (spread blockage), in order to exclude possible long term transients, simulations are run until $t=4000$.

We begin with the case where there is no stepping stone and the size of the passage is makes it impassable. One such parameter set was identified in simulations as

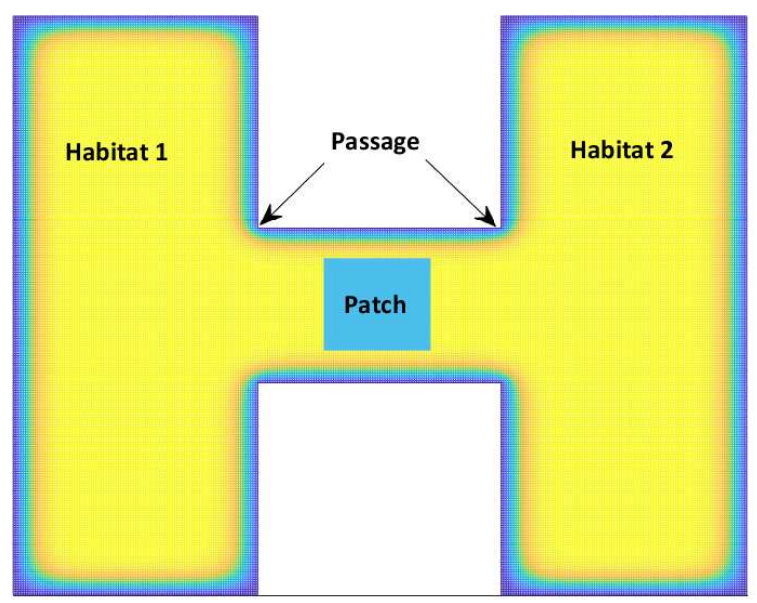

Figure 9: The H-shaped domain with the stepping stone located inside the passage. 
(a) $t=10$

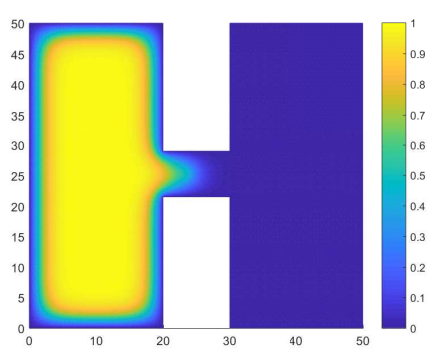

(b) $t=500$

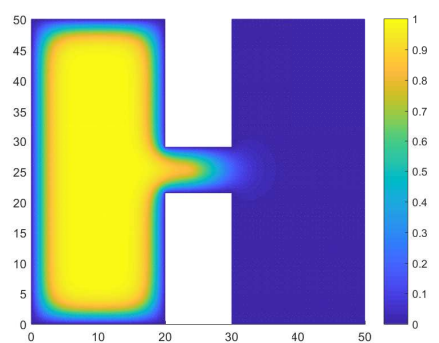

(c) $t=4000$

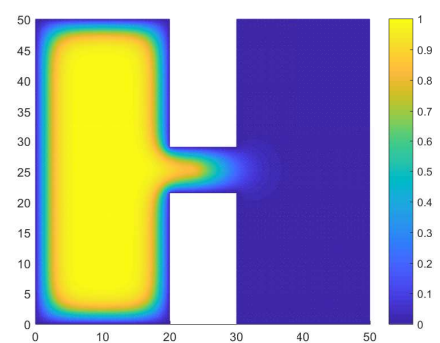

Figure 10: Snapshots of the population distribution over space obtained for the passage size as $($ length, width $)=(10,7.5)$, patch size of $2 \times 2$ and the patch location $l_{p}=2.8$.

(length,width $)=(10,7.5)$. With the passage of this size, the alien species is blocked and never proliferates into Habitat 2. It is intuitively clear that a small stepping stone is unlikely to unblock the passage, and this is indeed what is seen in simulations. Figure 10 shows the results obtained in the case where there is a $2 \times 2$ patch with $\beta_{w}=-0.5$ located at the distance $l_{p}=2.8$ from the passage entrance. It is readily seen that, for this patch size and location, the population spread remains blocked by the passage.

Interestingly, a patch with the same parameters as above but at different location inside the passage can lead to different dynamics changing invasion failure to invasion success. Figure 11 show the results obtained in the case where $l_{p}=2.9$, i.e. the patch is located just slightly further away from the passage entrance (other parameters are the same as in Fig. 10). It is readily seen that in this case the passage is unblocked and the population fast spreads to Habitat 2.

In order to provide a comprehensive overview of the system's properties it is therefore necessary to consider the dynamics subject to different parameter combinations. This has been achieved by means of extensive numerical simulations varying all relevant parameters over a broad range. Essential results are shown in Figs. 12-13. Figure 12 shows the map in parameter plane $\left(\beta_{w}, l_{p}\right)$ obtained for several different sizes of the stepping stone patch. It is readily seen that, the larger the patch size is, the broader is the range of parameters where invasion is successful. For the patch size of $5 \times 5$ or larger, invasion blocking is only

(a) $t=100$

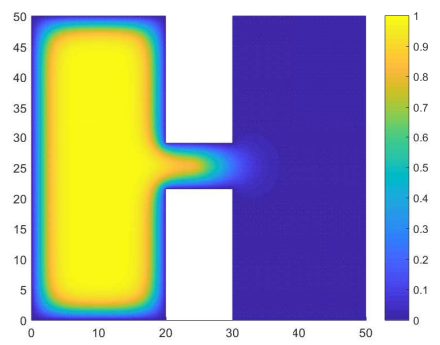

(b) $t=300$

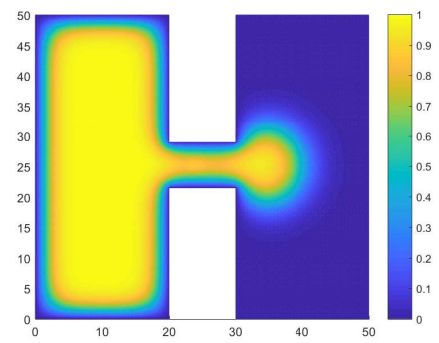

(c) $t=400$

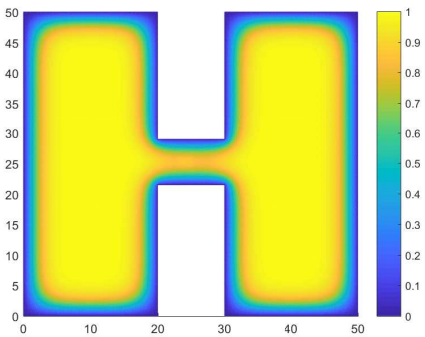

Figure 11: Snapshots of the population distribution over space obtained for the patch location $l_{p}=2.9$, other parameters are the same as in Fig. 10. 
possible for a few particular combination of parameter values such as values of $\beta_{w}$ close to zero and the patch location close to the either passage entrance (i.e. close to Habitat 1) or to the passage exit (close to Habitat 2), see, respectively, the bottom-right and the top-right corners in Fig. 12d. For the stepping stone patch of a small size, see Figs. 12a and $12 \mathrm{~b}$, invasion can only be successful if the patch located approximately in the middle of the passage but not at the entrance to the passage. Note that, unless the patch size is large, e.g. equal to or larger than one half of the passage width (cf. Fig. 12c), an increase in $\beta_{w}$ will always result in invasion blocking, even that the Allee effect in the patch is still weak and the conditions are favourable.

We want to mention that, for the parameters of Fig. 12, the passage without the stepping stone would block the spread regardless of the strength of the Allee effect inside the patch; see Fig. 9a in [2]. Therefore, the existence of the stepping stone can unblock the otherwise impassable passage.

It is readily seen from Fig. 12 that, for the passage of a fixed size, the parameter domain corresponding to successful invasion (cf. light-blue colored areas in Fig. 12) grows steadily with an increase in the stepping stone patch size. When the patch size approaches the width of the passage, there parameter range where invasion can be blocked shrinks

(a) Patch size $1.5 \times 1.5$

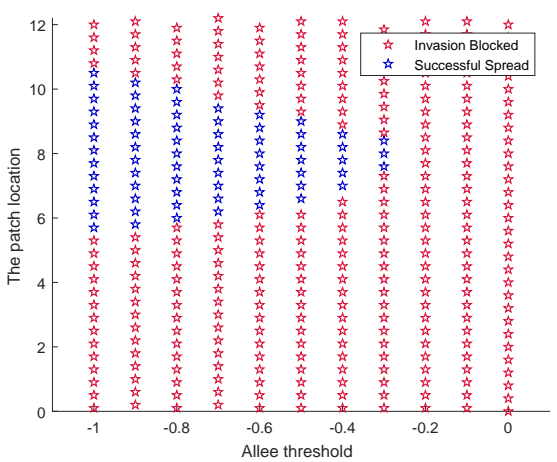

(c) Patch size $3.5 \times 3.5$

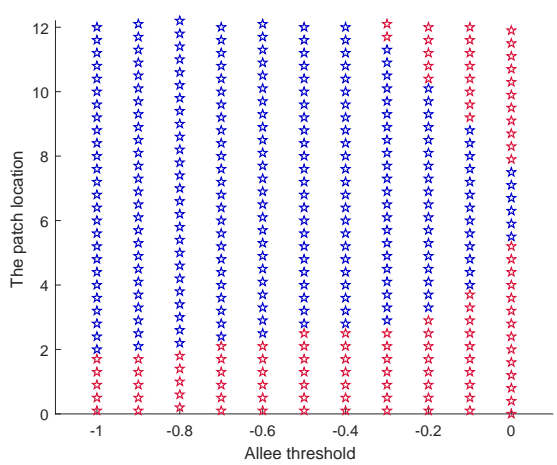

(b) Patch size $2 \times 2$

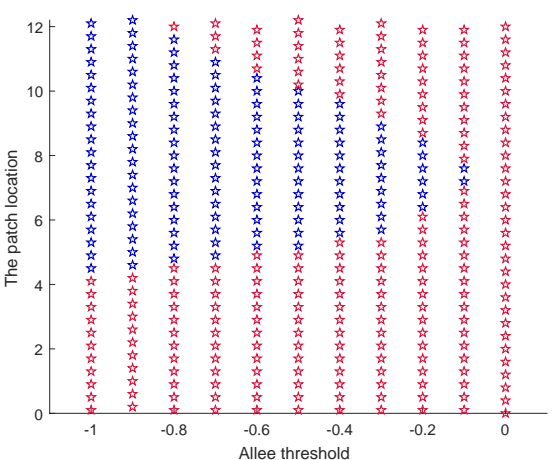

(d) Patch size $4.5 \times 4.5$

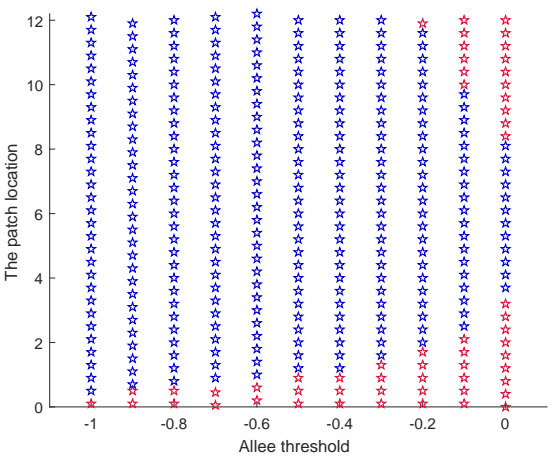

Figure 12: Map in parameter plane $\left(\beta_{w}, l_{p}\right)$ showing parameter ranges where the invasion is successful (blue color) and parameter ranges where invasion is blocked by the passage (red color) for the stepping stones of different size. Parameters of the passage are (length, width $)=(12,7.5)$. 
(a) Passage size $=(12,7.6)$

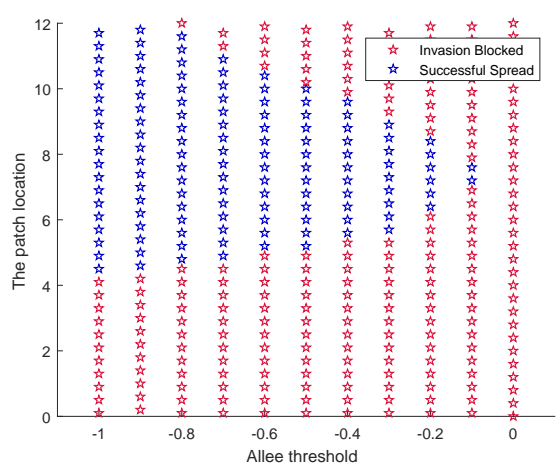

(c) Passage size $=(9,6.6)$

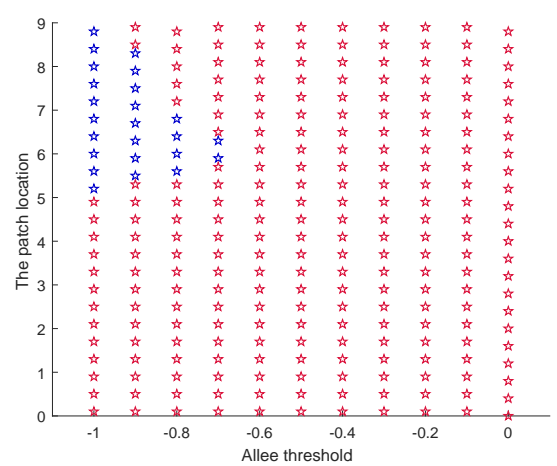

(b) Passage size $=(10,7)$

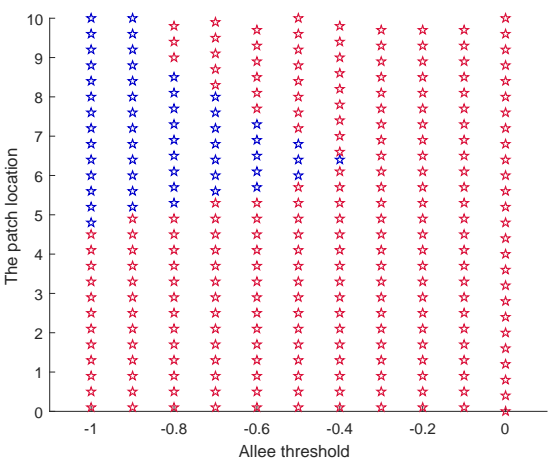

(d) Passage size $=(8,6.2)$

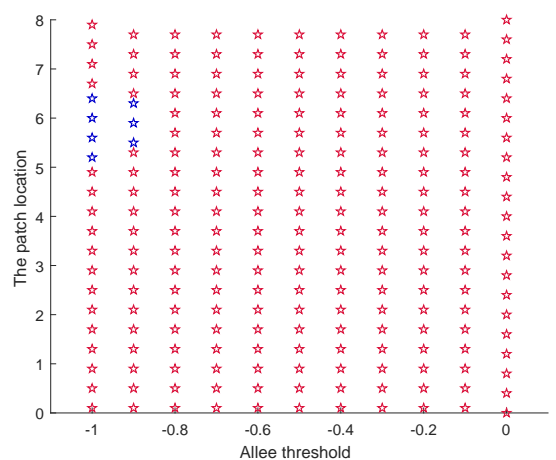

Figure 13: Map in parameter plane $\left(\beta_{w}, l_{p}\right)$ showing parameter ranges where the invasion is successful (blue color) and parameter ranges where invasion is blocked by the passage (red color) obtained for the stepping stone $2 \times 2$ and the passage of different size.

to zero. A question can arise as to whether the system's dynamics actually depends on the ratio of the passage width and the patch size rather than on these two parameters separately. In order to address this question, we fix the size of the stepping stone patch as $2 \times 2$ and perform simulations with the passage of different sizes. The results are shown in Fig. 13. It is readily seen that the parameter domain of successful invasion shrinks along with a decrease in the passage width, hence showing the tendency opposite to that observed in Fig. 12.

\subsection{Effects of different growth rate normalization}

The above results were obtained under the assumption that normalizing coefficient $\hat{\gamma}$ in Eq. (2.2) (or coefficient $\gamma$ in original Eq. (2.1)) is constant, i.e. not related to any other species traits. Although this assumption is routinely used in mathematical ecology $[19,62,85,91]$, a closer look reveals that it is in fact not well justified biologically. Indeed, it is readily seen that in this case a change in the value of threshold density $\beta$ leads to a change in the maximum growth rate and the maximum per capita growth rate [55]. Meanwhile, the properties of the population growth rate at small population densities 
(where the Allee effect is especially important) and at intermediate population densities (where the maximum growth rate is reached) are often determined by different factors and processes and hence are not necessarily directly related. Therefore, as an alternative to the simple case $\hat{\gamma}=1$, now we are going to consider a model where these processes are uncoupled. That requires normalizing coefficient to become a function of the Allee threshold, i.e. $\hat{\gamma}=\hat{\gamma}(\beta)$, where the particular function depends on what property is being preserved.

Namely, we consider the following two cases [55]:

- The maximum growth rate is kept constant (i.e. independent of $\beta$ ). The corresponding expression for the normalizing coefficient is as follows:

$$
\hat{\gamma}=27 /\left(2\left(\left((1+\beta)^{2}-9 \beta / 2\right)(1+\beta)+\left((1+\beta)^{2}-3 \beta\right)^{\frac{3}{2}}\right)\right) .
$$

- The maximum per capita growth rate is kept constant; the corresponding expression for $\hat{\gamma}$ is

$$
\hat{\gamma}=4 /(1-\beta)^{2}
$$

We now perform simulations using the 2D model (3.1), (3.3) with $\hat{\gamma}(\beta)$ given by either (3.4) or (3.5) in the H-shaped domain with a stepping stone in the passage; see Fig. 9. Results are summarized in Figs. 14-15.

Figure 14 shows parameter plane $\left(\beta_{w}, l_{p}\right)$ of the stepping stone parameters obtained in the case of scaling (3.4) preserving the maximum growth rate. The size of the passage is $($ length,width $)=(5,2.6)$. We first check that without the stepping stone this passage is impassable for the population (see also [2]). The presence of the stepping stone assists the spread and can unblock the passage. It is readily seen from Fig. 14 that, although the shape of the map is somewhat different from that obtained in the previous section,

(a) Patch size $1.6 \times 1.6$

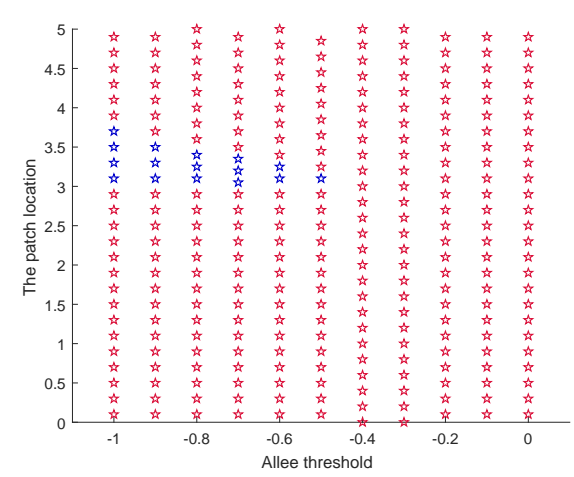

(b) Patch size $1.9 \times 1.9$

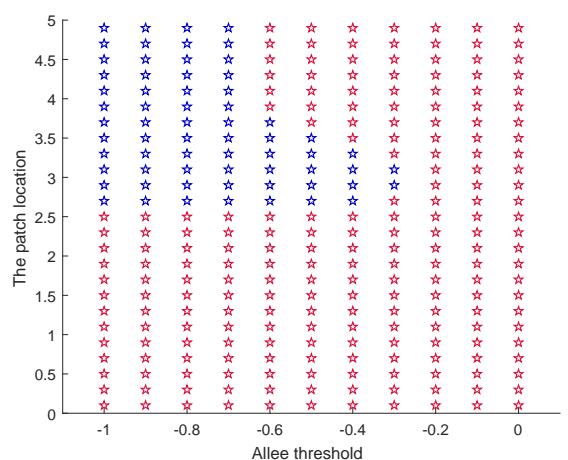

Figure 14: Map in parameter plane $\left(\beta_{w}, l_{p}\right)$ showing parameter ranges where the invasion is successful (blue color) and parameter ranges where invasion is blocked by the passage (red color) for the stepping stones of different size and the normalizing coefficient $\hat{\gamma}$ chosen according to Eq. (3.4). Parameters of the passage are (length, width $)=(5,2.6)$. 
(a) Patch size $1.6 \times 1.6$

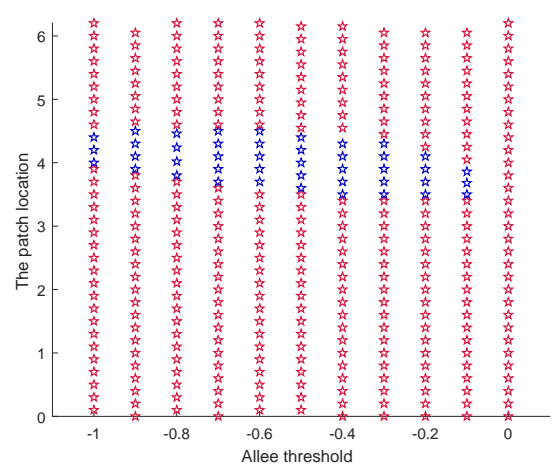

(b) Patch size $2.2 \times 2.2$

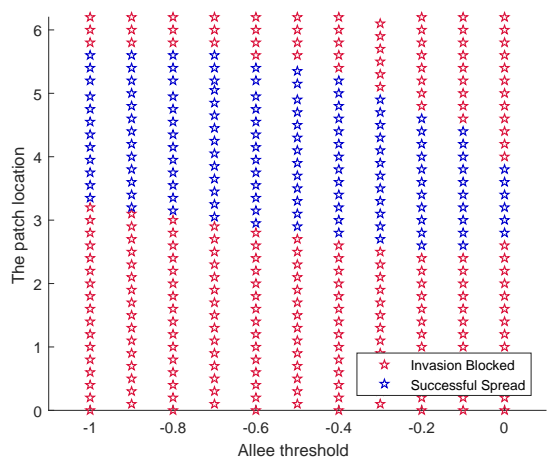

Figure 15: Map in parameter plane $\left(\beta_{w}, l_{p}\right)$ showing parameter ranges where the invasion is successful (blue color) and parameter ranges where invasion is blocked by the passage (red color) for the stepping stones of different size and the normalizing coefficient $\hat{\gamma}$ chosen according to Eq. (3.5). Parameters of the passage are (length, width) $=(5,3.4)$.

the general tendencies remain essentially the same as in the case of $\hat{\gamma}=1$. The existence of a stepping stone can unblock the otherwise impassable passage; the larger the stepping stone is, the more distinct this effect is (i.e. the broader the corresponding parameter range is). For the stepping stone of a small size (cf. Fig. 14a), it can only unblock the passage if it is located at a certain optimum location approximately in the middle of the passage.

Figure 15 shows parameter plane $\left(\beta_{w}, l_{p}\right)$ obtained for the stepping stones of different size in the case where $\hat{\gamma}$ is given by Eq. (3.5) (hence preserving the maximum per capita growth rate). The size of the passage is (length, width $)=(5,3.4)$; note that without the stepping stone this passage is impassable [2]. As well as above, we observe that the stepping stone can assist the spread by unblocking the passage. For smaller patches, there is a certain optimum location but a larger patch unblocks the passage in a broader range of parameters.

\section{Discussion and concluding remarks}

Biological invasion has been a focus of intense research for several decades because of severe consequences that alien species' spread can cause to biodiversity, environment, forestry and agriculture [20, 25, 43, 70, 78, 92]. Once a new species is introduced and starts spreading, evaluation of the extent of the invadable area becomes an important issue. It is often addressed by means of species distribution modelling (SDM) which attempts to predict the future distribution of invasive species on the basis of the known distribution in their native range by revealing the correlation between the values of the population density (often only the presence/abence data) and relevant environmental parameters such as temperature, precipitation, soil type, land cover, etc. [4]. However, 
the capability of SDM to correctly predict the extent of the actually invaded area depends on various factors that can affect species dispersal, such as barriers. Meanwhile, it is well known that dispersal is a limiting factor that affects species distribution; moreover, under certain unfavorable conditions (e.g. under the global climate change) restricted dispersal can endanger species survival altogether [81]. In other words, SDM generally succeeds (subject to the quality of available data) to identify the potential areas where the alien species can establish and persist, but it says very little about how the alien species will actually get there and whether it will get there at all.

Heterogeneity of the environment often reduces the space accessible to alien species spread to narrow corridors [88]. In conservation programs, corridors are generally regarded as a landscape feature that promotes species persistence [32]. In the context of the invasive spread, however, their effect is different as they tend to slow down the spread [3, 29] or even can block it altogether [2]. Although empirical research into the effect of corridors on invasive species spread remains meagre (arguably, because of considerable technical and methodological difficulties [29]), its tendency to hamper the spread was demonstrated in modelling studies using different models, e.g. see [2, 3, 29].

On the other hand, the importance of stepping stones for species dispersal has long been recognised [7, 40]. It is usually considered as a feature promoting long-distance dispersal [79], i.e. dispersal on a large spatial scale (such as is given, for instance, by an archipelago of small islands [79]). In our study, we endeavored to consider the effect of stepping stones on a much smaller spatial scale by linking them to dispersal corridors. We considered a situation where the corridor connecting two large habitats has got a small area or patch inside that has more favourable conditions than the rest of the environment. In agreement with previous studies, we found that stepping stones promote species spread; the larger is the stepping stone patch, the more noticeable its effect is. However, we also found that their actual efficiency to promote the alien species spread depends significantly on the relation between three factors such as their size, the quality of the environment inside the patch (quantified here as the strength of the Allee effect) and their location. In particular, we found that there exists an optimum location of the stepping stone where even a stepping stone of small size and with relatively poor environmental conditions can unblock the otherwise impassable corridor.

A question may arise here with regard to the generality of our findings. Indeed, in our study we considered the effect of the stepping stones in the particular case of rectangular geometry (as is given by the 'H-shaped' domain) and with the zero-function Dirichlet boundary conditions at the boundaries of the passage. In order to check the generality of our findings with regard to the domain shape, we considered the population dynamics described by Eq. (3.1) in a domain with a different 'keyhole' geometry; see Fig. 16. We performed a simulation study similar to Section 3.2 with the same aim to reveal how the existence of a favorable patch in the passage (i.e. in the central field) can affect the spread of the invading species from Habitat 1 on the left to Habitat 2 on the right. Interestingly, our finding were essentially the same as above. In particular, we 


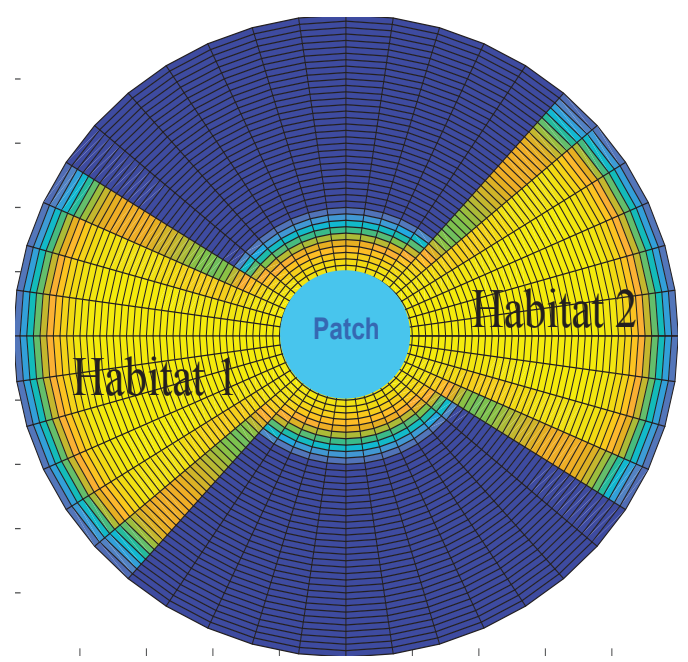

Figure 16: A sketch of the 2D computational domain with polar symmetry with a stepping stone patch (an area with better conditions, i.e. a weaker Allee effect) inside the central field.

observed that the existence of the stepping stone assists the species spread through the otherwise impassable passage. For a small patch, the passage can only be unblocked if the stepping stone is situated at the narrow range of locations (in this case, at the entrance to Habitat 2). For a larger patch, this range of locations grows fast, so that for a sufficiently weak Allee effect inside the patch the stepping stone unblocks the passage at almost any location. Arguably, this apparent similarity between the results obtained for two different geometries suggests that our main conclusions are not too sensitive to the shape of the domain.

With regard to different conditions at the passage boundary, the species spread in the H-shaped domain without a stepping stone was considered in [3]. It was shown there that the spread through the passage can be blocked if the passage is sufficiently narrow but the dynamical mechanisms resulting in the spread blocking and, correspondingly, the conditions of blocking are different for the Dirichlet (zero-function) and the Neumann (zero-flux) conditions at the passage boundaries; see Section 3.1 in [3]. Whilst in the case of Dirichlet conditions the spread is blocked in the middle of the passage, in the case of Neumann conditions the spread is blocked at the exit to Habitat 2. Correspondingly, the effect of the stepping stone is slightly different. In order to unblock the impassable passage, in the case of Neumann conditions the stepping stone must be located inside Habitat 2, not inside the passage. This is readily seen from the heuristic arguments and confirmed by numerical simulations (not shown here for the sake of brevity). Apart from this subtle difference, the effect of the stepping stone in the Neumann case is similar to that in the Dirichlet case: it promotes the spread, the range of stepping stone locations where it unblocks the passage is narrow for a small patch but grows fast with an increase in the patch size.

In this paper, we have considered the effect of habitat's boundaries in the context of 
landscape geometry, i.e. aiming to understand how the shape of natural landscape features can affect the spread of invasive species. However, we mention here that a similar problem arises in relation to invasive species control. Indeed, one way to control the spread used in practical applications is to create landscape features - "barriers" - inaccessible for the invader $[83,84]$. Arguably, the effect of the boundaries of those human-made structures on the dynamics of the invading population is going to be the same as the effect of natural landscape structures, so that our analysis is fully relevant. Our results therefore may help to provide a more efficient design of the barriers.

\section{References}

[1] M.W. Adamson, and A. Y. Morozov. When can we trust our model predictions? Unearthing structural sensitivity in biological systems. Proc. R. Soc. A. The Royal Society, 469 (2012), 20120500.

[2] Alharbi WG, Petrovskii SV, 2016. The impact of fragmented habitats size and shape on populations with Allee effect. Mathematical Modelling of Natural Phenomena $11(4), 5-15$.

[3] Alharbi, W.G., Petrovskii, S.V. (2018) Patterns of invasive species spread in a landscape with a complex geometry. Ecol. Compl. 33, 93-105.

[4] Araujo MB, Guisan A., 2006. Five (or so) challenges for species distribution modelling. J. Biogeogr. 33, 1677-1688.

[5] D. J. Bailey, W. Otten, C. A. Gilligan (2000) Saprotrophic invasion by the soilborne fungal plant pathogen Rhizoctonia solani and percolation thresholds. The New Phytologist 146, 535-544.

[6] Barenblatt GI, 1996. Scaling, self-similarity, and intermediate asymptotics. Cambridge: Cambridge University Press.

[7] Baum, K.A., Haynes, K.J., Dillemuth, F.P. \& Cronin, J.T. (2004) The matrix enhances the effectiveness of corridors and stepping stones. Ecology 85, 2671-2676.

[8] J. Benitez-Malvido and V. Arroyo-Rodriguez. Habitat fragmentation, edge effects and biological corridors in tropical ecosystems. Encyclopaedia of Life Support Systems (EOLSS), Oxford: Eolss Publishers, (2008), 1-11.

[9] Bergelson, J., Newman, J.A., Floresroux, E.M. (1993) Rates of weed spread in spatially heterogeneous environments. Ecology 74, 999-1011. 
[10] M. Bevers and C. H. Flather. Numerically exploring habitat fragmentation effects on populations using cell-based coupled map lattices. Theor. Popul. Biol., 55(1) (1999), 61-76.

[11] P. Cheptou, V. Avendano, and G. Lyz. Pollination processes and the Allee effect in highly fragmented populations: consequences for the mating system in urban environments. New Phytol., 172(4) (2006), 774-783.

[12] S. K. Collinge. Ecological consequences of habitat fragmentation: implications for landscape architecture and planning. Landsc. Urban Plan., 36(1) (1996), 59-77.

[13] F. Courchamp, T. Clutton-Brock, and B. Grenfell. Inverse density dependence and the Allee effect. Trends Ecol. Evol., 14(10) (1999), 405-410.

[14] Franck Courchamp, Alice Fournier, Cline Bellard, Cleo Bertelsmeier, Elsa Bonnaud, Jonathan M. Jeschke, James C.Russell (2017) Invasion Biology: Specific Problems and Possible Solutions. Trends Eecol. Evol. 32, 13-22.

[15] J. Crank. The Mathematics of Diffusion. Oxford University Press, 1979.

[16] de-Camino-Beck, T., Lewis, M.A.: Invasion with stage-structured coupled map lattices: application to the spread of scentless chamomile. Ecol. Model. 220(23), 33943403 (2009).

[17] B. Dennis. Allee effects: population growth, critical density, and the chance of extinction. Nat. Resour. Model., 3(4) (1989), 481-538.

[18] J. B. Dunning, B. J. Danielson, and H. R. Pulliam. Ecological Processes that Affect Populations in Complex Landscapes. Oikos, 65(1) (1992), 169-175.

[19] L. Edelstein-Keshet. Mathematical Models in Biology. SIAM, 1988.

[20] Elton, C.S.: The Ecology of Invasions by Animals and Plants. Methuen, London (1958)

[21] R. M. Ewers and R. K. Didham. Confounding factors in the detection of species responses to habitat fragmentation. Biol. Rev., 81(01) (2006), 117-142.

[22] L. Fahrig. Effects of habitat fragmentation on biodiversity. Annu. Rev. Ecol. Evol. Syst., 34 (2003), 487-515.

[23] J. Fischer and D.B. Lindenmayer. Landscape modification and habitat fragmentation: a synthesis. Glob. Ecol. Biogeogr., 16(3) (2007), 265-280.

[24] C. Flora, N. David, G. Mathias, A. Morozov, andP. Jean-Christophe. Structural sensitivity of biological models revisited. J. Theor. Biol., 283(1) (2011), 82-91. 
[25] Drake, J.A., Mooney, H.A., di Castri, F., Groves, R.H., Kruger, F.J.: Biological Invasions: A Global Perspective. Wiley, Chichester (1989)

[26] Farlow, S.J. Partial Differential Equations for Scientists and Engineers; Dover: New York, NY, USA, 1993.

[27] G.F. Fussmann, and B. Blasius. Community response to enrichment is highly sensitive to model structure. Biol. Lett., 1(1) (2005), 9-12.

[28] Garcia-Ojalvo, J. and J. M. Sancho (1999). Noise in spatially extended systems. Institute for Nonlinear Science. New York: Springer.

[29] Gardner, R. H., Forester, J. D., \& Plotnick, R. E. (2007). Determining pattern process relationships in heterogeneous landscapes. In Key Topics in Landscape Ecology (ed. by J. Wu, R.J. Hobbs), pp. 92-114. Cambridge University Press.

[30] Garvie M.R., Burkardt J., Morgan J. (2015) Simple finite element methods for approximating predator-prey dynamics in two dimensions using MATLAB. Bull. Math. Biol. 77, 548-578.

[31] Garvie M.R., Morgan J., Sharma V. (2017) Finite element approximation of a spatially structured metapopulation PDE model. Comp. Math. Applic. 74, 934-947.

[32] Gibbs JP, 1998. Amphibian movement in response to forest edges, roads, and streambeds in Southern New England. J. Wildlife Management 62, 584-589.

[33] Giometto, A., Altermatt, F., Rinaldo, A. (2017) Demographic stochasticity and resource autocorrelation control biological invasions in heterogeneous landscapes. Oikos 126, 1554-1563.

[34] D. Goodman. Consideration of stochastic demography in the design and management of biological reserves. Nat. Resour. Model., 1 (1987a), 205-234.

[35] I. Hanksi and M. Gilpin. Metapopulation dynamics: brief history and conceptual domain. Biol. J. Linn. Soc., 42(1-2) (1991), 3-16.

[36] Holmes, E.E., Lewis, M.A., Banks, J.E., Veit, R.R.: Partial differential equations in ecology: spatial interactions and population dynamics. Ecology 75, 1729 (1994)

[37] Jankovic, M., Petrovskii, S.V. (2013) Gypsy moth invasion in North America: a simulation study of the spatial pattern and the rate of spread. Ecol. Compl. 14, 132-144.

[38] M. Jankovic and S. Petrovskii. Are time delays always destabilizing? Revisiting the role of time delays and the Allee effect. Theor. Ecol., 7(4) (2014), 335-349. 
[39] Johnson, D.M., Liebhold, A.M., Tobin, P.C., Bjrnstad, O.N.: Allee effects and pulsed invasion by the gypsy moth. Nature 444(7117), 361363 (2006).

[40] Jopp, F., and H. Reuter. 2005. Dispersal of carabid beetles: emergence of distribution patterns. Ecological Modelling 186, 389-405.

[41] Keitt, T.H., Lewis, M.A., Holt, R.D.: Allee effects, invasion pinning, and species borders. Am. Nat. 157, 203216 (2001).

[42] Keitt, T., D. L. Urban and B. T. Milne 1997. Detecting Critical Scales in Fragmented Landscapes. Conservation Ecology 1(1):4.

[43] Keller, R.P., Lodge, D.M., Lewis, M.A., Shogren, J.F.: Bioeconomics of Invasive Species: Integrating Ecology, Economics, Policy, and Management. Oxford University Press, Oxford (2009).

[44] H. Kierstead and L.B. Slobodkin. The size of water masses containing plankton blooms. J. mar. Res., 12(1) (1953), 141-147

[45] R. V. Kirk and M. A. Lewis. Edge permeability and population persistence in isolated habitat patches. Nat. Resour. Model., 12(1) (1999), 37-64.

[46] Kot, M.: Discrete-time travelling waves: ecological examples. J. Math. Biol. 30, 413436 (1992).

[47] M. Kot. Elements of Mathematical Ecology. Cambridge University Press, 2001.

[48] Kot M., Lewis, M.A. \& van der Driessche, P. 1996 Dispersal data and the spread of invading organisms. Ecology 77, 2027-2042.

[49] W. Kunin and Y. Iwasa. Pollinator foraging strategies in mixed floral arrays: density effects and floral constancy. Theor. Popul. Biol., 49(2) (1996), 232-263.

[50] B. B. Lamont, P. G. Klinkhamer, and E. Witkowski. Population fragmentation may reduce fertility to zero in Banksia goodiia: demonstration of the Allee effect. Oecologia, 94(3) (1993), 446-450.

[51] R. Lande. Risks of population extinction from demographic and environmental stochasticity and random catastrophes. Am. Nat., 142(6) (1993), 911-927.

[52] R. Lande. Extinction thresholds in demographic models of territorial populations. Am. Nat., 130(4) (1987), 624-635.

[53] W. F. Laurance and E. Yensen. Predicting the impacts of edge effects in fragmented habitats. Biol. Conserv., 55(1) (1991), 77-92.

[54] Lewis, M.A.: Spread rate for a nonlinear stochastic invasion. J. Math. Biol. 41, 430454 (2000). 
[55] M. Lewis and P. Kareiva. Allee dynamics and the spread of invading organisms. Theor. Popul. Biol., 43(2) (1993), 141-158.

[56] Lewis, M.A., Pacala, S.: Modeling and analysis of stochastic invasion processes. J. Math. Biol. 41, 387429 (2000).

[57] Lewis, M.A., Petrovskii, S.V., and Potts, J. (2016) The Mathematics Behind Biological Invasions. Interdisciplinary Applied Mathematics, Vol. 44. Springer.

[58] G. J. Lord, V. Thümmler (2012) Computing stochastic traveling waves. SIAM J. Sci. Comput., 34(1), B24-B43.

[59] D.C. Mistro, L.A.D. Rodrigues \& S.V. Petrovskii (2012) Spatiotemporal complexity of biological invasion in a space- and time-discrete predator-prey system with the strong Allee effect. Ecol. Compl. 9: 16-32.

[60] A.Y. Morozov and B.L. Li. On the importance of dimensionality of space in models of space-mediated population persistence. Theor. Popul. Biol., 71(3) (2007), 278-289.

[61] D. Moser, H. G. Zechmeister, C. Plutzar, N. Sauberer, T. Wrbka, and G. Grabherr. Landscape patch shape complexity as an effective measure for plant species richness in rural landscapes. Landsc. Ecol., 17(7) (2002), 657-669.

[62] Murray, J.D., 2001. Mathematical Biology II: Spatial Models and Biomedical Applications. Interdisciplinary Applied Mathematics. Springer, New York.

[63] Neubert, M.G., Kot, M., Lewis, M.A.: Dispersal and pattern-formation in a discretetime predatorprey model. Theor. Popul. Biol. 48, 743 (1995).

[64] Owen, M.R. \& Lewis, M.A. 2001 How predation can slow, stop or reverse a prey invasion. Bull. Math. Biol. 63, 655-684.

[65] Petrovskii, S.V. (1997) Localization of a nonlinear switching wave in an active medium with an isolated inhomogeneity. Technical Physics 42, 866-871.

[66] Petrovskii, S.V. (1998) Modelling of open-sea ecological impact: impact wave localization and pattern formation. Environment Modelling and Assessment 3, 127-133.

[67] S. V. Petrovskii and B. Li. Exactly Solvable Models of Biological Invasion. CRC Press, 2005.

[68] S. V. Petrovskii and B. Li. An exactly solvable model of population dynamics with density-dependent migrations and the Allee effect. Math. Biosci., 186(1) (2003), 79-91.

[69] Petrovskii, S.V., Morozov, A.Y., Venturino, E. (2002) Allee effect makes possible patchy invasion in a predator-prey system. Ecology Letters 5, 345-352. 
[70] Pimentel, D. (ed.): Biological Invasions: Economic and Environmental Costs of Alien Plant, Animal, and Microbe Species. CRC Press, Boca Raton (2002).

[71] B. J. Rathcke. Habitat fragmentation and plant pollinator. Curr. Sci. , 65(3) (1993), 273-277.

[72] Renardy, M.; Rogers, R.C. An Introduction to Partial Differential Equations; Springer: New York, NY, USA, 2006.

[73] Richardson, D.M. (ed.): Fifty Years of Invasion Ecology: The Legacy of Charles Elton. Wiley, Chichester (2011).

[74] Richardson, D.M., Pysek, P.: Fifty years of invasion ecology: the legacy of Charles Elton. Divers. Distrib. 14(2), 161168 (2008).

[75] N. Richter-Dyn and N. S. Goel. On the extinction of a colonizing species. Theor. Popul. Biol., 3(4) (1972), 406-433.

[76] L. Ries and T. D. Sisk. A predictive model of edge effects. Ecology, 85(11) (2004), 2917-2926.

[77] Sakai, A.K., Allendorf, F.W., Holt, J.S., Lodge, D.M., Molofsky, J., With, K.A., Baughman, S., Cabin, R.J., Cohen, J.E., Ellstrand, N.C., McCauley, D.E., ONeil, P., Parker, I.M., Thompson, J.N., Weller, S.G.: The population biology of invasive species. Annu. Rev. Ecol. Syst. 32, 305332 (2001).

[78] Sandlund, O.T., Schei, P.J., Viken, A.: Invasive Species and Biodiversity Management. Kluwer Academic, Dordrecht (2001)

[79] Saura S, Bodin O, Fortin M-J, 2014. Stepping stones are crucial for species longdistance dispersal and range expansion through habitat networks. J. Appl. Ecol. 51, 171-182.

[80] D. W. Schemske, B. C. Husband, M. H. Ruckelshaus, C. Goodwillie, I. M. Parker, and J. G. Bishop. Evaluating approaches to the conservation of rare and endangered plants. Ecology, 75(3) (1994), 584-606.

[81] Schloss, C. A. et al., 2012. Dispersal will limit ability of mammals to track climate change in the Western Hemisphere. PNAS 109, 8606-8611.

[82] Seebens, H., Gastner, M.T., Blasius, B.: The risk of marine bioinvasion caused by global shipping. Ecol. Lett. 16, 782790 (2013).

[83] Sharov A A, Liebhold A M (1998) Model of slowing the spread of gypsy moth (Lepidoptera: Lymantriidae) with a barrier zone. Ecological Applications 8(4):1170-1179. 
[84] Sharov A A, Leonard D, Liebhold A M, Roberts E A, Dickerson W (2002) "Slow the Spread": A national program to contain the gypsy moth. Journal of Forestry 100(5): 30-36(7).

[85] N. Shigesada \& K. Kawasaki (1997) Biological Invasions: Theory and Practice. Oxford: Oxford University Press.

[86] J. G. Skellam. Random dispersal in theoretical populations. Biometrika, 38(1/2) (1951), 196-218.

[87] C. Thies and T. Tscharntke. Landscape structure and biological control in agroecosystems. Science, 285 (5429) (1999), 893-895.

[88] Reid Tingley, Benjamin L. Phillips, Mike Letnic, Gregory P. Brown, Richard Shine and Stuart J. E. Baird, 2013. Identifying optimal barriers to halt the invasion of cane toads Rhinella marina in arid Australia. Journal of Applied Ecology 50, 129-137.

[89] W. van Saarloos (2003) Front propagation into unstable states. Physics Reports 386, 29-222.

[90] V. Volpert and S. Petrovskii. Reaction-diffusion waves in biology. Phys. Life. Rev., 6(4) (2009), 267-310.

[91] M.-E. Wang, M. Kot, Speeds of invasion in a model with strong or weak Allee effects, Math. Biosci. 171 (2001) 83.

[92] Williamson, M.: Biological Invasions. Chapman \& Hall, London (1996). 